\title{
Variabilidade temporal do volume e caracterização física e química dos sedimentos do açude São José III no Cariri Paraibano
}

\section{Temporal variability of the volume and physical and chemical characterization of sediment weir São José III in Cariri Paraibano}

\author{
Danilo R. Monteiro', Tainara T. S. Silva ${ }^{2}$, Maria S. S. Farias ${ }^{3}$, Hallyson Oliveira , Felipe G. de Souza ${ }^{5}$
}

\begin{abstract}
Resumo: Esse trabalho teve como objetivo analisar os características físico-químicas do sedimento do Açude de São José III, situado no município de São José dos Cordeiros no estado da Paraíba, bem como a pluviometria da região e o volume do manancial nos últimos 5 anos. Realizou-se a coleta do sedimento no Açude São José III, em três pontos distintos (A-Fundo do manancial; B-Margem do açude - depósito de resíduos; C-Margem do açude-área agricultável) a uma profundidade de 0-30 cm com auxílio de trado manual, pás plásticas e tubos de PVC (50 mm de diâmetro) e acondicionadas em sacos plásticos. Após a coleta, as amostras passaram por secagem ao ar, destorroadas e enviadas ao Laboratório de Irrigação e Salinidade (LISUFCG), para realização das análises física e químicas. Afirma-se com os dados pluviométricos o ano que apresentou a maior média foi o de $2009(103,89 \mathrm{~mm})$ e o de menor foi o de $2012(16,59 \mathrm{~mm})$. A análise granulométrica dos sedimentos revelou que o sedimento da amostra A (Fundo do Manancial) possui 50,06\% de argila. As amostras de sedimento B (Margem do Manancial - Depósito de resíduos) e C (Margem do Manancial - Área agricultável) apresentaram uma fração de areia superior (B-80,74\%; C-62,64\%), na caracterização química percebeu-se que a razão C/N obtida nas amostras do açude São José III possuem origem de ambientes terrestres e aquáticos.
\end{abstract}

Palavras-chave: limnologia, granulometria, resíduos.

Abstract: This study has how to objective to analyze the physical and chemical characteristics of the sediment of São José III, located in São José dos Cordeiros - PB, as well as the rainfall of the region and the volume of the weir the last 5 years. Held the collection of the sediment weir São José III, at three different points (A-Fund of the weir, B-border of the weir - waste disposal; C-Border of the weir - arable area) to a depth of $0-30 \mathrm{~cm}$ with the support of manual auger, plastic blades and PVC tubes ( $50 \mathrm{~mm}$ diameter) and packed in plastic bags. After collect, the samples passed air drying, broken into pieces and sent to the Laboratory of Irrigation and Salinity (LIS-UFCG), to peform the physical and chemical analyzes. Says with the rainfall data for the year with the highest average was $2009(103.89 \mathrm{~mm})$ and the lowest was observed in 2012 (16.59 $\mathrm{mm})$. The particle size analysis of the sediment revealed that the sediment sample A (Fund of the weir) has 50.06\% argil. Sediment samples B (Border of the weir - waste disposal) and C (Border of the weir - arable area) showed a higher sand fraction (B-80, $74 \%, \mathrm{C}-62,64 \%)$, realized that the chemical characterization to $\mathrm{C} / \mathrm{N}$ ratio in the samples of the weir São José III have origin of terrestrial and aquatic environments.

Keywords : limnology, granulometry.

\footnotetext{
*Autor para correspondência

Recebido para publicação em 21/08/2014; aprovado em 01/05/2015

${ }^{1}$ Engenheiro Agrícola, Universidade Federal de Campina Grande - UFCG

${ }^{2}$ Engenheira Agrícola, Doutoranda em Engenharia Agrícola Universidade Federal de Campina Grande - UFCG

${ }^{3}$ Professora Dra. Unidade Acadêmica de Engenharia Agrícola. Universidade Federal de Campina Grande - UFCG.

${ }^{4}$ Engenheiro Agrícola, Mestrando em Engenharia Agrícola Universidade Federal de Campina Grande - UFCG

${ }^{5}$ Engenheiro Agrícola, Mestrando em Engenharia Agrícola Universidade Federal de Campina Grande - UFCG
} 


\section{INTRODUÇÃO}

O uso indiscriminado do solo e dos recursos hídricos para os mais variados fins, apontam para a importância do estudo da quantificação, no tempo e no espaço, dos impactos causados nesses ambientes. Fatores importantes que podem ser levados em consideração são a produção, transporte e deposição de sedimentos nos corpos hídricos.

$\mathrm{O}$ aumento do sedimento de fundo e dos sedimentos em suspensão em rios, lagos e represas, pode estar relacionado à incidência de chuvas em regiões que sofreram alguma ação antrópica, a exemplo da supressão da cobertura vegetal, aplicação indiscriminada de praguicidas ou herbicidas e deposição de resíduos em locais inadequados. Estes fatos corroboram para o carreamento do solo e contaminação dos mananciais, pela deposição dessas partículas transportadas.

De modo geral os sedimentos podem ser definidos como um aglomerado de partículas minerais e orgânicas que, interagem com a parte inferior dos corpos de água naturais (BAIRD, 2011), fornecendo subsídios para a variação de diversos organismos e corroboram para a avaliação e quantificação da intensidade dos impactos no ambiente aquático (BATISTA, 2015). Dessa forma, os sedimentos constituem-se em fontes de contaminação primária para os organismos bentônicos e secundária para a coluna d'água (ADAMS, 1992).

O estudo do tamanho das partículas (granulometria) que constitui os sedimentos em conjunto com a matéria orgânica existente, possibilita tecer informações importantes sobre relações entre o habitat e os organismos bentônicos, qualidade de água e os possíveis impactos do manancial em estudo.

Diversos elementos podem ser transportados em drenagens basicamente de duas formas: espécies dissolvidas na água ou associadas a partículas sólidas (SANTOS, 2008). Como partículas sólidas, eles podem ocorrer tanto na parte da estrutura cristalina ou na mineral adsorvida na superfície da argila (POPP, 1998). Ao contrário daqueles na estrutura cristalina, os elementos adsorvidos nas argilas estão fisicamente ligados ao substrato, de tal forma que os mesmos são mais reativos que os primeiros e, portanto, possuem uma maior disponibilidade (SMITH; HUYCK, 1999).

As frações granulométricas mais importantes nas discussões sobre a contaminação por substâncias químicas são a argila e o silte. Essas partículas de menor tamanho apresentam maior potencial de adsorção de metais (BRIGANTE; ESPÍNOLA, 2003). Ao serem erodidas e transportadas da bacia de drenagem para os corpos hídricos reagem com os contaminantes existentes e com os transportados para o ambiente aquático (SOUSA, 2010).

O conhecimento da concentração de sedimentos é importante para avaliação das consequências de intervenção humana na bacia hidrográfica, no assoreamento de barragens, para o caso de estuários no estudo de escoamento de canais de acesso a barcos de acesso e berços de atracação em portos (BANDEIRA; AUN, 1989). Partindo desse pressuposto o presente trabalho teve como foco principal fazer o levantamento do volume do Açude de São José III para o período de 2009-2014 e a avaliação físico-química dos sedimentos, visando gerar informações que venham a contribuir com a gestão dos recursos hídricos na bacia hidrográfica do Rio Paraíba.

\section{MATERIAL E MÉTODOS}

\section{Característica da área em estudo}

O estudo foi desenvolvido na cidade de São José dos Cordeiros-PB (07 $23^{\circ}$ ' 27''S, 36 $46^{\circ}$ ' $28^{\prime}$ ' $\mathrm{W}$ ), altitude $610 \mathrm{~m}$ ) na região fisiográfica dos Cariris Velhos. O município possui uma área de $417,74 \mathrm{~km}^{2}$ e população de 3.985 habitantes (IBGE, 2010). A precipitação média da região é 550mm (600 a $800 \mathrm{~mm} / \mathrm{ano}$ ) e o clima da região, segundo a classificação de Koeppen, é do tipo BShsemiarido quente com chuvas de verão (ATLAS GEOGRÁFICO DO ESTADO DA PARAÍBA, 1985).

A coleta do material sedimentar foi realizada no Açude São José III, este apresenta uma capacidade total de $956.000 \mathrm{~m}^{3}$, fazendo parte da Bacia Hidrográfica do rio Paraíba, sub-bacia do rio Taperoá. Os solos da região são do tipo Brunos não cálcicos, LitolíticosEutróficos, Solos aluviais, Regosol (AESA,2014)-(Figura 1).

Figura 1: Açude São José III (local de coleta de material), bacia hidrográfica onde está inserido o manancial e mapa de solos da Paraíba.

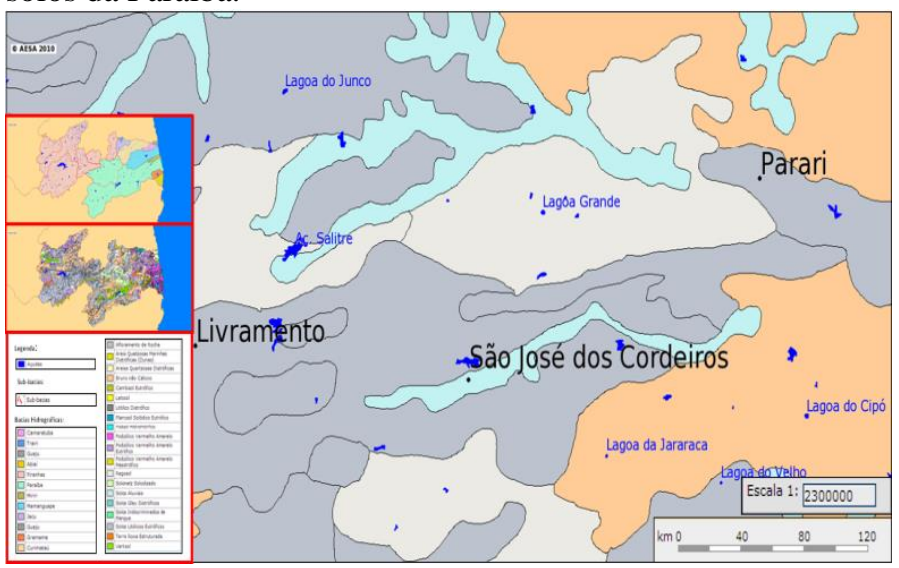

Fonte: AESA (2014).

\section{Levantamento das precipitações e do volume do açude}

Para avaliação temporal referente ao período de 2009 a 2014 das precipitações e volumes do manancial, foi realizada uma pesquisa no banco de dados da AESA (Agência Executiva de Gestão das águas do Estado da Paraíba).

\section{Coleta das amostras do sedimento do açude}

As amostras de sedimentos foram coletadas em três pontos distintos (A-Fundo do mancial; B-Margem do açude depósito de resíduos; C-Margem do açude-área agricultável) a uma profundidade de $0-30 \mathrm{~cm}$. Os pontos foram escolhidos levando-se em consideração a profundidade máxima do manancial (A), as margens do açude onde se localiza um depósito de resíduos sólidos urbanos (B) e a margem mais agricultável quando do período de chuva (C).

Para coleta das amostras utilizou-se de trado manual (tipo caneco com $100 \mathrm{~mm}$ de diâmetro), pás plásticas e tubos de PVC (50 mm de diâmetro) e acondicionadas em sacos plásticos.

Após a coleta as amostras passaram por secagem ao ar, destorroadas e enviadas ao Laboratório de Irrigação e Salinidade (LIS-UFCG), para realização das análises física 
e químicas de acordo com metodologia proposta pela EMBRAPA(1997).

\section{Variáveis analisadas}

As variáveis químicas e físicas (Granulometria,), Cálcio, Carbono Orgânico, Nitrogênio Total, Matéria Orgânica, Fósforo e pH) foram analisadas de acordo com metodologia proposta por EMBRAPA (1997).

\section{RESULTADOS E DISCUSSÃO}

Análise Pluviométrica e do Volume do Açude São José III

Observa-se nos dados de pluviometria mensal e do volume do Açude São José III dos últimos 5 anos contidos na Figura 3, que durante esse período o manancial esteve em sua capacidade máxima em dois anos, em 2009 e 2011, onde passou 5 meses e 4 meses sangrando, respectivamente. Em 2012, mais precisamente entre os meses agosto e dezembro o açude entrou em colapso, se repetindo o fato no ano de 2013. No referido ano o corpo hídrico permaneceu impróprio para o consumo, apresentando um volume insuficiente para atender a demanda. Em 2014, o açude atingiu o colapso total, permanecendo durante dois meses (Janeiro e Fevereiro) totalmente seco.

Ainda segundo a Figura 2, observa-se com os dados pluviométricos o ano que apresentou a maior média foi o de $2009(103,89 \mathrm{~mm})$ e o de menor foi o de $2012(16,59 \mathrm{~mm})$. Segundo Carvalho (1994) as chuvas que desagregam o solo e as enxurradas são os principais responsáveis pelo transporte de sedimentos para os cursos d'água

Figura 2. Gráfico de pluviometria total e volume total do açude de são José III (2009-2014).

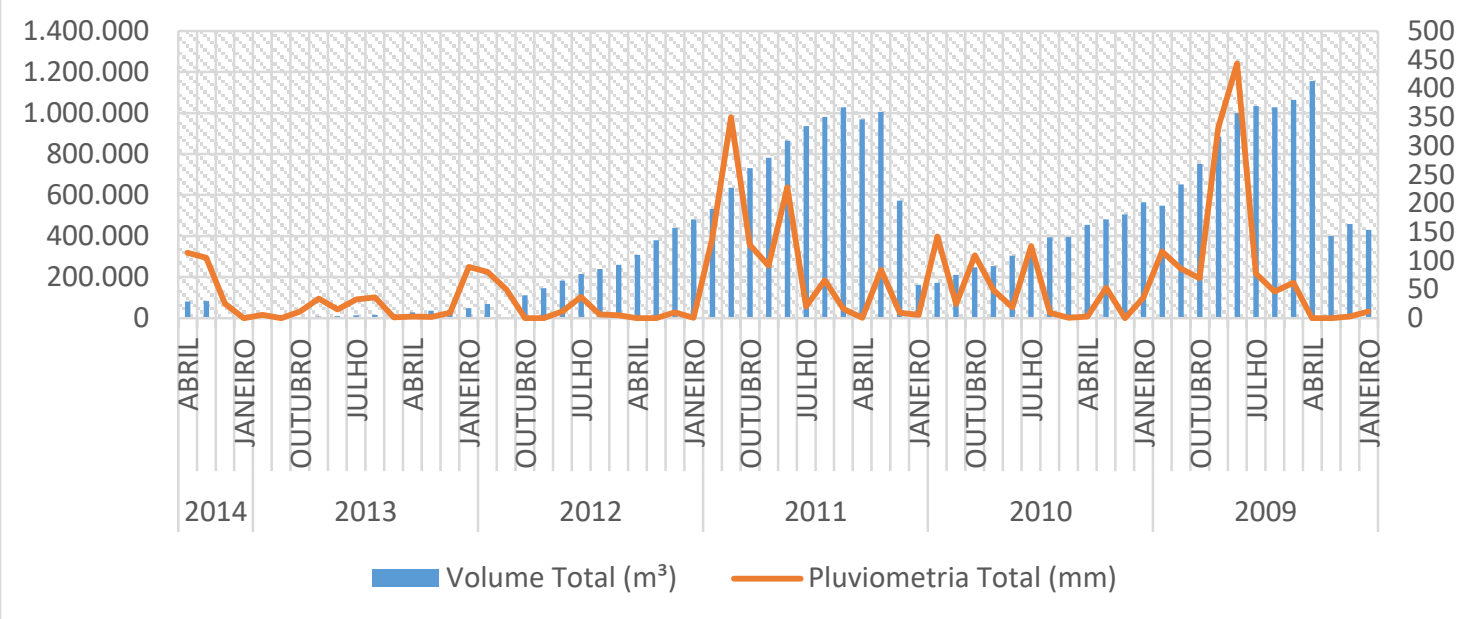

Fonte: AESA (2014).

Quando o material transportado por um curso d'água provém da superfície da bacia contribuinte ele é caracterizado como deflúvio (Washload) e seu transporte se dá predominantemente em suspensão, atingindo em torno de 90 a 95\% do total dos sedimentos transportados (SHEN, 1971; EINSTEIN, 1964; UMEZAWA, 1979; FIGUEIREDO, 1989).

Num diagnóstico sedimentológico realizado na bacia do rio São Francisco, Carvalho (1995) mostrou que o aumento da produção de sedimentos está sendo causado pela urbanização e mineração na bacia, pelo aumento de áreas agrícolas para a produção de alimentos, pela necessidade de construção de estradas, aliados a presença de fortes chuvas. Na região estudada nunca foi realizado uma recuperação do manancial para retirada dos sedimentos do fundo com objetivo de recuperar o volume antes planejado, comprometendo a oferta de água para região, vale salientar que na área do entorno fica um deposito de lixo que contribui para aumentar a poluição local.

\section{Características Físicas}

\section{Granulometria do Sedimento}

A análise granulométrica dos sedimentos (Tabela 1) indica que o sedimento da amostra A (Fundo do Manancial) possui
$50,06 \%$ de argila mais $22,46 \%$ de Silte, sendo classificada texturalmente como Argila. Isso aponta para o risco do manancial sofrer com a contaminação por substâncias químicas, visto que metais se associam referencialmente às frações finas dos sedimentos (silte e argila).

Segundo Kato e Piveli (2005) observaram que quanto menor o tamanho da partícula maior será a sua relação com a área superficial e volume. Concordando com Forster e Solomons (1980) que ao estudarem traços de metais em sedimentos poluídos perceberam que a fração fina (silte e argila) apresentam maior teor de elementos traços devido a sua superfície de contato.

As amostras de sedimento nos pontos B (Margem do Manancial - Lixão) e C (Margem do Manancial - Área agricultável) apresentaram uma fração de areia superior (B80,74\%; C-62,64\%), sendo classificadas texturalmente como Areia Franca e Franco Arenoso, respectivamente.

Amostras com frações granulométricas arenosas, demonstram a possibilidade de efeito erosivo da região marginal no açude. Nas Figuras 3A e 3B observa-se as frações granulométricas maiores que sofreram transporte da bacia de drenagem até o açude. 
Tabela 1: Análise granulométrica do sedimento e classificação textural.

\begin{tabular}{|c|c|c|c|c|}
\hline \multirow{2}{*}{ Pontos de Coleta } & \multicolumn{3}{|c|}{ Granulometria (\%) } & \multirow{2}{*}{$\begin{array}{l}\text { Classificação } \\
\text { Textural }\end{array}$} \\
\hline & Areia & Silte & Argila & \\
\hline A (Fundo do Manancial) & 27,48 & 22,46 & 50,06 & Argila \\
\hline $\begin{array}{l}\text { B (Margem do Manancial - Depósito } \\
\text { de resíduos) }\end{array}$ & 80,74 & 10,12 & 9,14 & Areia Franca \\
\hline $\begin{array}{l}\text { C (Margem do Manancial - Área } \\
\text { agricultável) }\end{array}$ & 62,64 & 18,16 & 19,2 & Franco Arenoso \\
\hline
\end{tabular}

Figura 3: A- Efeito erosivo causado pelo deslocamento de partícula de diâmetro maior; B- Frações granulométricas carreadas da bacia de drenagem até o manancial

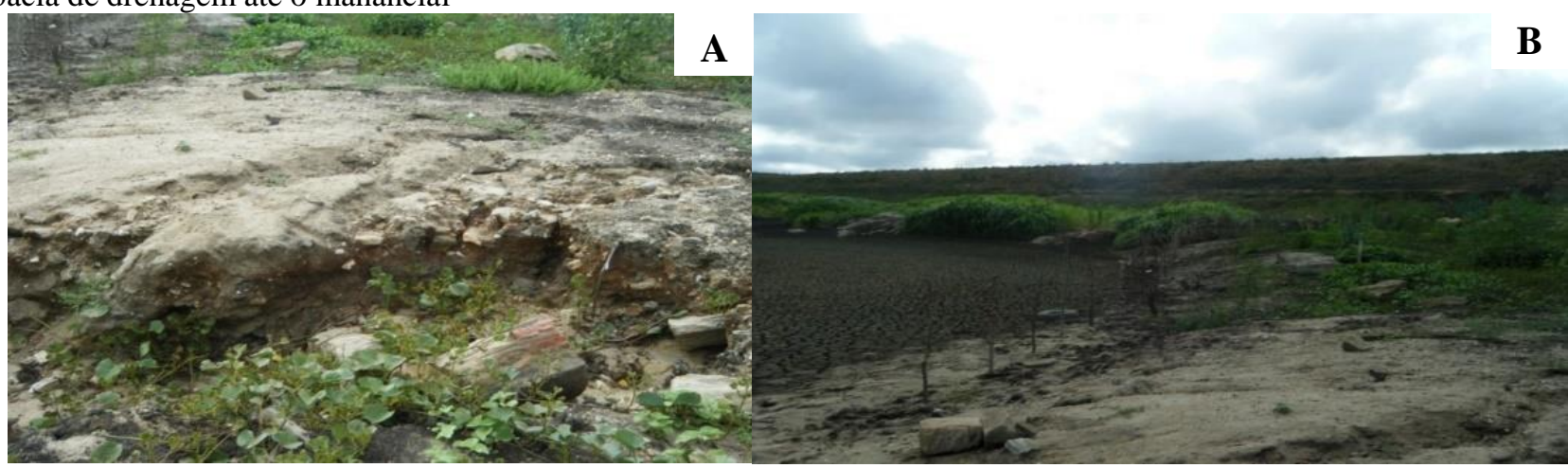

De um modo geral pode-se dizer que a composição granulométrica dos sedimentos é um dos fatores responsáveis pela estrutura e distribuição das comunidades biológicas que habitam os mananciais (CALLISTO; ESTEVES, 1996; GONÇALVES et al., 1998). Além disso de forma secundária a qualidade da água também é afetada pela deposição sedimentar.

\section{Características Químicas}

\section{Carbono Orgânico e Nitrogênio total e a origem da} matéria orgânica

Analisar os constituintes da matéria orgânica permite identificar o intervalo de maior produtividade de Carbono orgânico $\left(\mathrm{C}_{\mathrm{org}}\right)$ e Nitrogênio total $\left(\mathrm{N}_{\text {tot }}\right)$ e identificar a origem da matéria orgânica através da relação $\mathrm{C} / \mathrm{N}$.

Essa relação tem sido usada para diferenciar fontes terrestres e aquáticas de matéria orgânica (STEIN, 1991; ANDREWS et al., 1998; KANELLOPOULLOS et al., 2006).

Os elementos constituintes da matéria orgânica analisados são o carbono e o nitrogênio, podendo ser observados em percentagem na Tabela 2 .

Tabela 2:Conteúdo de Carbono Orgânico $\left(\mathrm{C}_{\mathrm{org}}\right)$, Nitrogênio total $\left(\mathrm{N}_{\text {tot }}\right)$ e relação $\mathrm{C} / \mathrm{N}$ a uma profundidade de $0-30 \mathrm{~cm}$ de sedimento do Açude São José III.

\begin{tabular}{|c|c|c|c|}
\hline Pontos de Coleta & $\begin{array}{c}\text { Carbono } \\
\text { Orgânico (\%) }\end{array}$ & $\begin{array}{l}\text { Nitrogênio Total } \\
(\%)\end{array}$ & $\begin{array}{c}\mathrm{C} / \mathrm{N} \\
(\mathrm{MOLAR})\end{array}$ \\
\hline A (Fundo do Manancial) & 1,95 & 0,19 & 10,26 \\
\hline B (Margem do Manancial - Depósito de resíduos) & 0,63 & 0,06 & 10,5 \\
\hline C (Margem do Manancial - Área agricultável) & 0,74 & 0,07 & 10,57 \\
\hline
\end{tabular}

$\mathrm{O}$ aumento do aporte de matéria orgânica pode ser justificado pelo aumento nos teores de $\mathrm{C}_{\text {org }}$ e $\mathrm{N}_{\text {tot }}$. Isso foi evidenciado principalmente nos setores de amostragem que apresentaram intervenções antropogênicas, como agricultura e depósito de resíduos. Esse mesmo fato foi observado por Santos (2010), onde testemunhos de sedimentos do açude Marcela em Sergipe apresentaram maiores valores para concentração de $\mathrm{C}_{\mathrm{org}}$ e $\mathrm{N}_{\mathrm{tot}}$ em profundidades inferiores a 30 $\mathrm{cm}$.

A origem da matéria orgânica no sedimento de lagos, rios e oceanos tem sido avaliada por indicadores como o conteúdo de $\mathrm{C}_{\text {org }}$ e $\mathrm{N}_{\text {tot. }}$. Através de razões envolvendo esses indicadores, é possível discutir sobre as fontes prováveis na composição da matéria orgânica (SANTOS, 2010). A razão $\mathrm{C} / \mathrm{N}$ determinada a partir de teores percentuais de $\mathrm{C}_{\text {org }}$ e $\mathrm{N}_{\text {tot }}$ tem sido usada na distinção entre matéria orgânica originada de algas e bactérias e matéria orgânica originada de plantas terrestres (MERYES, 1997; RUTTENBERG; GUÑI, 1997; ANDREWS et al., 1998; KANELLOPOULOS et al., 2006).

A matéria orgânica de origem animal possui mais proteína que os vegetais terrestres, enquanto as algas possuem teores de proteínas superiores maiores que os das plantas terrestres. Assim a razão $\mathrm{C} / \mathrm{N}$ de organismos planctônicos varia entre 4,0 e 7,0 (SANTOS, 2010), os bentônicos têm valores para a razão próximos a 4,0 (MULLER; MATHESIUS, 1999) e as bactérias entre 4,0 e 6,0 (RUTTENBERG; GOÑI, 1997).

Segundo Kanellopoulos et al (2006) a relação C/N entre 6,6 e 10, apresentada em sedimentos, tem origem da matéria orgânica derivada de ambientes terrestres e do próprio ambiente aquático. Assim sendo, podemos afirmar que a 
razão C/N obtida nas amostras do açude São José III possuem origem de ambientes terrestres e aquáticos.

\section{Matéria Orgânica}

A matéria orgânica é um dos principais componentes dos sedimentos pois interfere nos processos químicos, físicos e biológicos, sendo determinante nas reações de síntese secundária (húmificação). As substâncias húmicas, resultantes do processo de humificação, possuem uma capacidade de interagir com metais gerando complexos de diferentes estabilidades e características estruturais (SOUSA, 2010).

De acordo com o teor de matéria orgânica o sedimento pode ser classificado como orgânico ou inorgânico. Segundo Esteves (1998) para ser considerado orgânico o sedimento deve conter mais de $10 \%$ de matéria orgânica em seu peso seco, e será considerado inorgânico quando o teor for menor que $10 \%$. Para os valores obtidos nas amostras coletadas no Açude São José III e suas margens, conclui-se que o sedimento é classificado como inorgânico.

$\mathrm{Na}$ Tabela 3 são apresentados os valores da matéria orgânica das amostras coletadas no manancial. O ponto $\mathrm{A}$ (Fundo do Manancial) foi o que apresentou maior percentual de matéria orgânica $(3,36 \%)$. $\mathrm{O}$ que pode ser relacionado ao fato de que a fração granulométrica do ponto A é argila, assim ocorrem processos abióticos de adsorção e complexação química com óxidos de alumínio (BRIGANTE \& ESPÍNOLA et al., 2003).

Tabela 3: Percentagem de matéria orgânica do sedimento do açude são José III e suas margens.

\begin{tabular}{lc}
\hline Pontos de Coleta & Matéria Orgânica (\%) \\
\hline A (Fundo do Manancial) & 3,36 \\
B (Margem do Manancial - Depósito de resíduos) & 1,08 \\
C (Margem do Manancial - Área agricultável) & 1,27 \\
\hline
\end{tabular}

\section{Fósforo, Cálcio e pH.}

Do ponto de vista liminológico o íon fosfato (P-orto), assume a maior relevância por ser a principal forma de fosfato assimilada pelos vegetais aquáticos. Desta maneira, a sua quantificação em pesquisas limnológicas torna-se indispensável (ESTEVES, 1998).

Na Tabela 4 estão expostos a quantidade de fósforo assimilável. O ponto A (Fundo do Manancial) apresentou a menor taxa de P-orto $(1,09 \mathrm{mg})$ e o ponto B (Margem do
Manancial - Depósito de Resíduos) apresentou a maior taxa de P-orto, correspondendo a 2,10 mg.

Possivelmente o menor teor de fósforo apresentado no ponto A se deve a mais alta concentração de íons Cálcio, dentre as amostras coletadas, causando um processo denominado de precipitação de hidroxiapatita, em condições de altos valores de $\mathrm{pH}$.

Tabela 4:Teor de Fósforo, Cálcio e pH em amostras do sedimento do Açude de São José III.

\begin{tabular}{|c|c|c|c|}
\hline Pontos de Coleta & Fósforo (mg $\left.100 \mathrm{~g}^{-1}\right)$ & $\begin{array}{l}\text { Cálcio (mmol } \\
\left.100 \mathrm{~g} \text { de } \text { solo }^{-1}\right)\end{array}$ & pH $\mathrm{H}_{2} \mathrm{O}(1: 2,5)$ \\
\hline A (Fundo do Manancial) & 1,09 & 5,63 & 8,00 \\
\hline $\begin{array}{l}\text { B (Margem do Manancial - Depósito de } \\
\text { resíduos) }\end{array}$ & 2,10 & 1,54 & 8,44 \\
\hline $\begin{array}{l}\text { C (Margem do Manancial - Área } \\
\text { agricultável) }\end{array}$ & 2,08 & 3,40 & 8,20 \\
\hline
\end{tabular}

\section{CONCLUSÃO}

A análise da fração granulométrica apontou para o risco de contaminação do manancial, a partir da deposição dos sedimentos transportados.

As áreas marginais do manancial apresentaram efeito erosivo.

A razão Carbono Nitrogênio apontou que a matéria orgânica é derivada de ambientes terrestres e aquáticos.

O sedimento é classificado como Inorgânico.

No sedimento de fundo ocorre o fenômeno denominado de precipitação de hidroxiapatita.

\section{REFERÊNCIAS}

AESA- Agência Executiva de Gestão das Águas do Estado da Paraíba. http://www.aesa.pb.gov.br, acesso em: 08 de Junho de 2014.

ADAMS, W.J. Aquatic toxicology testing methods. In: Hoffman, D.J.; Rattner, B.A.; Burton Jr., G.A.; Cairns Jr., J. (eds). Handbook of ecotoxicology. Lewis Publisker - CRC Press. 1995, p. 25-46.

ANDREWS, J. E; GREENAWAY, A. M.; DENNIS, P. F. Combined Isotope and $\mathrm{C} / \mathrm{N}$ Ratios as Indicators of Source on Fate of Organic Matter in a Poorly Flushed, Tropical Estuary: Hunts Bay, Kingston Harbour, Jamaica. Estruarine, CoastalandShelf Science, v.46, p.743 - 756, 1998.

Atlas Geográfico do Estado da Paraíba, 1985. Secretaria da Educação. João Pessoa. 100 p.

BANDEIRA, J. V. \& AUN, P. E. Anais do VIII Simpósio Brasileiro de Recursos Hídricos. São Paulo. ABRH, 2v, 1989. p361.

BAIRD, C. Química ambiental. $4^{\circ}$ ed. Porto Alegre: Bookman, 2011.

BATISTA, M. A. R. da S. Avaliação da qualidade da água e sedimento de microbacias utilizadas para abastecimento 
público da região metropolitana de Goiânia. Tese de Doutorado. 2015.

BRIGANTE, J. \& ESPÍNOLA, E. L. G.;Limnologia fluvial: um estudo do rio Mogi-Guaçu. Rima, São Carlos, 2003. 278p.

CALLISTO, M.; ESTEVES, F.A. Composição granulométrica do sedimento de um lago Amazônico impactado por rejeito de bauxita e um lago natural (Pará, Brasil). Acta LimnologicaBrasiliensia, v. 8, p. 115-126, 1996.

CARVALHO, N. Erosão Crescente na Bacia do São Francisco. Revista Brasileira de Engenharia, Caderno de Recursos Hídricos. Vol. 13, Nº 2, Dezembro/1995. p. 5-19.

CARVALHO, N. Hidrossedimentologia Prática. Rio de Janeiro. CPRM,1994. 372 p.

EINSTEIN, H. A. 1964. River Sedimentation. In: Chow, VemTe, ed. Handbook of applied hydrology. New York, McGraw-Hill. Sect. 17 - II, pt. 2.

EMBRAPA - Empresa Brasileira de Pesquisa Agropecuária. Manual de métodos de análise do solo. 2. ed. Rio de Janeiro: EMBRAPA - Centro Nacional de Pesquisa de Solos. p. 247, 1997.

ESTEVES, F. Fundamentos da Limnologia. $2^{\mathrm{a}}$ ed. Interciência, Rio de Janeiro, 602p. 1998.

FIGUEIREDO, A. G. Análise da produção e transporte de sedimentos nas bacias do rio do Peixe e rio Aguapeí. Anais do VIII Simpósio Brasileiro de Recursos Hídricos. São Paulo. ABRH, 2v, 1989.

FÖRSTNER, U.; W. SALOMONS, Trace metal analysis on polluted sediments part I. Assessment of sources and intensities. Envir. Technol. Lett. v. 1, p. 494-504, 1980.

GONÇALVES, J.F. Jr.; CALLISTO, M.; FONSECA, J.J. Relações entre a composição granulométrica do sedimento e as comunidades de macroinvertebrados bentônicos naslagoasImboassica, Cabiúnas e Comprida (Macaé, RJ). In: ESTEVES, F. (Org.). Ecologia das lagoas costeiras do Parque Nacional da Restinga de Jurubatiba e do Município de Macaé, RJ. Rio de Janeiro: UFRJ, 1998. p. 299-310.

IBGE- Instituto Brasileiro de Geografia e Estatística. http://cidades.ibge.gov.br/xtras/perfil.php?lang=\&codmun=25 1480\&search=paraiba|sao-jose-dos-cordeiros, Acesso em: 08 de Maio de 2013.

KANELLOPOULOS, T. D.; ANGELIDIS, M. O.; KARAGEORGIS, A. P.; KABERI, H.; KAPSIMALIS, V.; ANAGNOSTOU, C. Geochemical composition of the uppermost prodelta sediments of the Evros River, northeastern Aegean Sea. Journal of Marine Systems v. 63, n. 1-2, p. 63-78, 2006.

MEYERS. Preservation of elemental and isotopic source indentification of sedimentary organic matter. Chemical Geology, v. 114, p. 289-302, 1997.

MULLER, A; MATHESIUS, U. The paleoenvironments of coastal lagoons in the southern Balic Sea, I. The application of sedimentary $\mathrm{C}_{\text {org }} / \mathrm{N}$ ratios as source indicators os organic matter. Paleo, v. 145, p.1--16, 1999.

POPP, J.H. Geologia Geral. Ed. JC.5 ${ }^{a}$ edição. Rio Grande do Sul-RS. 1998.

RUTTEMBERG, K. C.; GOÑI, M. A. Phosphorus distribution, $\mathrm{C}: \mathrm{N}: \mathrm{P}$ ratios, and $13 \mathrm{Coc}$ in arctic, temperate and tropical coastal sediments: tools for characterizing bulk sedimentary organic matter. Mar. Geol. v. 139, p. 123-145, 1997.

SANTOS, I. S. dos; Geoquímica e distribuição dos metaistraço em testemunhos de sedimento do alude Marcela, Itabaiana - Sergipe. 2010. Dissertação de Mestrado. 123 p.

SANTOS, A.R.L.; MELO Jr., G.; Segundo, J.E. de A. G. Concentração de metais pesados em frações granulométricas de sedimentos de fundo do Rio Pitimbu, região sul da grande Natal (RN): Implicações para levantamento ambiental. Revista de Geologia. 2002. V. 15, p.01-08.

SHEN, H. W. Wash load and bed load. River mechanics, v. 1, p. 11-1, 1971.

SMITH, K.S.; HUYCK, H.L.O. 1999. Na overview of the abundance, relative hability, bioaviability and human toxicity of metal. In: G.S. Plumlee\&M.J. Longdson (Ed) The Environmental Geochemistry of Mineral Deposits Part a: Processes, Techniques and Health Issues, Littleton, Society of Economic Geologist, Vol 6A. 29-70

SOLOMONS, W.; FORSTNER V. 1984. Metal in the Hidrocycle, New York. Springer-Verlag, 312p.

SOUSA, R. do R de; Caracterização química dos sedimentos superficiais de fundo do reservatório água preta (belém - pa). Programa de Pós- Graduação em Engenharia Civil da Universidade Federal do Pará. Dissertação de mestrado. 2010 ,

STEIN, R. Accumulation of organic matter in marine sediments. Results from deep Sea Drilling Project/ocean Drilling Program. In: Bhattacharji, S., Freidman, G.M. Neugebauer, H.J. Seilavher, A. eds, Lectures Notes in Eatrh Sciences. Berlin, Spring, 34.217P.1991.

UMEZAWA, P. K. Previsão de deplúvio (Washload) em rios de áreas elevadas. Porto Alegre, Universidade Federal do Rio Grande do Sul, Curso de Pós-Graduação em Recursos Hídricos e Saneamento. n. p. Dissertação de mestrado hidrologia aplicada. 1979. 217p. 